\title{
Pacific
}

Journal of

Mathematics

\section{EIGENVALUE ESTIMATES ON DOMAINS IN COMPLETE NONCOMPACT RIEMANNIAN MANIFOLDS}

Daguang Chen, Tao Zheng And Min Lu 


\title{
EIGENVALUE ESTIMATES ON DOMAINS IN COMPLETE NONCOMPACT RIEMANNIAN MANIFOLDS
}

\author{
Daguang Chen, Tao Zheng And Min Lu
}

\begin{abstract}
In this paper, we obtain universal inequalities for eigenvalues of the Dirichlet eigenvalue problem of the Laplacian and the clamped plate problem on a bounded domain in an $n$-dimensional $(n \geq 3)$ noncompact simply connected complete Riemannian manifold with sectional curvature Sec satisfying $-K^{2} \leq$ Sec $\leq-k^{2}$, where $K \geq k \geq 0$ are constants. When $M$ is $\mathbb{U}^{n}(-1)$ $(n \geq 3)$, these inequalities become ones previously found by Cheng and Yang.
\end{abstract}

\section{Introduction}

Let $M$ be an n-dimensional complete Riemannian manifold and $\Omega \subset M$ a bounded domain in $M$. The Dirichlet eigenvalue problem of the Laplacian is

$$
\begin{cases}\Delta u=-\lambda u & \text { in } \Omega \\ u=0 & \text { on } \partial \Omega .\end{cases}
$$

It is well known that the spectrum of this problem is real and discrete:

$$
0<\lambda_{1}<\lambda_{2} \leq \lambda_{3} \leq \cdots \nearrow \infty
$$

where each $\lambda_{i}$ has finite multiplicity which is repeated according to its multiplicity.

A Dirichlet eigenvalue problem of the biharmonic operator or a clamped plate problem that describes the characteristic vibrations of a clamped plate is given by

$$
\begin{cases}\Delta^{2} u=\Gamma u & \text { in } \Omega, \\ u=\frac{\partial u}{\partial v}=0 & \text { on } \partial \Omega,\end{cases}
$$

where $\Delta^{2}$ is the biharmonic operator on $M$ and $v$ denotes the outward normal derivative on $\partial \Omega$. We will denote eigenvalues and the corresponding real eigenfunctions by $\left\{\Gamma_{i}\right\}_{i=1}^{\infty}$ and $\left\{u_{i}\right\}_{i=1}^{\infty}$, respectively. The eigenvalues $\Gamma_{i}$ satisfy

$$
0<\Gamma_{1} \leq \Gamma_{2} \leq \Gamma_{3} \leq \cdots \nearrow \infty .
$$

D. Chen is partly supported by NSFC grant number 11101234 .

MSC2010: 35P15, 58J50, 58G25, 53C42.

Keywords: Laplacian, the Dirichlet problem, the clamped plate problem, eigenvalues, the universal inequality. 
When $M$ is a Euclidean space $\mathbb{R}^{n}$, these are estimates for the eigenvalues (1-1) that do not involve domain dependencies [Protter 1988]; see also [Ashbaugh 1999; 2002]. The main developments were obtained by Payne, Pólya, and Weinberger [Payne et al. 1956], Hile and Protter [1980], and Yang [1991]. More recently, for the Dirichlet eigenvalue problems of the Laplacian on a bounded domain in the $n$-dimensional unit sphere, complex projective space, and compact homogeneous Riemannian manifolds, Cheng and Yang [2005; 2006b; 2007] obtained the Yangtype inequalities for eigenvalues. For a bounded domain $\Omega$ in a complete Riemannian manifold $M$, the first author and Cheng [Chen and Cheng 2008] proved a Yang-type inequality by using the Nash embedding theorem (compare [El Soufi et al. 2009; Harrell 2007]).

By making use of estimates for eigenvalues of the eigenvalue problem of the Schröinger like operator with a weight, Harrell and Michel [1994], Ashbaugh [2002], and Ashbaugh and Hermi [2007] have obtained several results. In fact, for $n=2$, the Laplacian on $\mathbb{T}^{2}(-1)$ is like to the Laplacian on $\mathbb{R}^{2}$ with a weight. However, for $n>2$, this property does not hold. Cheng and Yang [2009] found appropriate trial functions and obtained

$$
\sum_{i=1}^{k}\left(\lambda_{k+1}-\lambda_{i}\right)^{2} \leq 4 \sum_{i=1}^{k}\left(\lambda_{k+1}-\lambda_{i}\right)\left(\lambda_{i}-\frac{(n-1)^{2}}{4}\right) .
$$

In this paper, we first treat the Dirichlet eigenvalue problem (1-1) of the Laplacian on a bounded domain of a complete noncompact Riemannian manifold $M$.

Theorem 1.1. Assume that $M^{n}(n \geq 3)$ is a noncompact simply connected complete Riemannian manifold with sectional curvature Sec satisfying $-K^{2} \leq \operatorname{Sec} \leq-k^{2}$, where $K \geq k \geq 0$ are constants.For a bounded domain $\Omega$ in $M$, let $\lambda_{i}$ be the $i$-th eigenvalue of the eigenvalue problem (1-1). Then we obtain

$$
\sum_{i=1}^{k}\left(\lambda_{k+1}-\lambda_{i}\right)^{2} \leq \sum_{i=1}^{k}\left(\lambda_{k+1}-\lambda_{i}\right)\left(4 \lambda_{i}-(n-1)^{2} k^{2}+2(n-1)\left(K^{2}-k^{2}\right)\right) .
$$

Remark. If $k=K=1$, that is, $M$ is a hyperbolic space $\mathbb{U}^{n}(-1)$, the eigenvalue inequality (1-4) agrees with (1-3) obtained by Cheng and Yang [2009].

The other purpose of this paper is to investigate estimates for eigenvalues of the clamped plate problem (1-2) on bounded domains $\Omega$ in a complete Riemannian manifold $M^{n}$.

For the universal inequalities for eigenvalues of the clamped plate problem in a bounded domain in $\mathbb{R}^{n}$, Payne et al. $[1955 ; 1956]$ proved that

$$
\Gamma_{k+1}-\Gamma_{k} \leq \frac{8(n+2)}{n^{2} k} \sum_{i=1}^{k} \Gamma_{i}, \quad k=1,2, \ldots
$$


Hile and Yeh [1984] obtained

$$
\sum_{i=1}^{k} \frac{\Gamma_{i}^{\frac{1}{2}}}{\Gamma_{k+1}-\Gamma_{i}} \geq \frac{n^{2} k^{3 / 2}}{8(n+2)}\left(\sum_{i=1}^{k} \Gamma_{i}\right)^{-\frac{1}{2}}, \quad k=1,2, \ldots
$$

Hook [1990] and Chen and Qian [1990] independently proved

$$
\frac{n^{2} k^{2}}{8(n+2)} \leq\left(\sum_{i=1}^{k} \frac{\Gamma_{i}^{\frac{1}{2}}}{\Gamma_{k+1}-\Gamma_{i}}\right)\left(\sum_{i=1}^{k} \Gamma_{i}^{\frac{1}{2}}\right), \quad k=1,2, \ldots
$$

Cheng and Yang [2006a] gave an affirmative answer for a problem on universal inequalities for eigenvalues proposed by Ashbaugh [1999]: they proved that

$$
\Gamma_{k+1}-\frac{1}{k} \sum_{i=1}^{k} \Gamma_{i} \leq\left(\frac{8(n+2)}{n^{2}}\right)^{\frac{1}{2}} \frac{1}{k}\left(\sum_{i=1}^{k} \Gamma_{i}\left(\Gamma_{k+1}-\Gamma_{i}\right)\right)^{\frac{1}{2}}, \quad k=1,2, \ldots
$$

For domains in a unit sphere, Wang and Xia [2007] gave a universal inequality for the clamped plate problem (1-2). They proved

$$
\sum_{i=1}^{k}\left(\Gamma_{k+1}-\Gamma_{i}\right)^{2} \leq \frac{8(n+2)}{n^{2}} \sum_{i=1}^{k}\left(\Gamma_{k+1}-\Gamma_{i}\right)\left(\Gamma_{i}^{\frac{1}{2}}+\frac{n^{2}}{2 n+4}\right)\left(\Gamma_{i}^{\frac{1}{2}}+\frac{n^{2}}{4}\right) .
$$

For an $n$-dimensional complete manifold $M$, Cheng, Ichikawa, and Mametsuka [Cheng et al. 2010] obtained

$$
\begin{aligned}
& \sum_{i=1}^{k}\left(\Gamma_{k+1}-\Gamma_{i}\right)^{2} \\
& \quad \leq \frac{8(n+2)}{n^{2}} \sum_{i=1}^{k}\left(\Gamma_{k+1}-\Gamma_{i}\right)\left(\Gamma_{i}^{\frac{1}{2}}+\frac{n^{2}}{2 n+4} \sup _{\Omega}|H|^{2}\right)\left(\Gamma_{i}^{\frac{1}{2}}+\frac{n^{2}}{4} \sup _{\Omega}|H|^{2}\right) .
\end{aligned}
$$

For the real hyperbolic space $\mathbb{H}^{n}(-1)$, Cheng and Yang [2011] proved that

$$
\sum_{i=1}^{k}\left(\Gamma_{k+1}-\Gamma_{i}\right)^{2} \leq 24 \sum_{i=1}^{k}\left(\Gamma_{k+1}-\Gamma_{i}\right)\left(\Gamma_{i}^{\frac{1}{2}}-\frac{(n-1)^{2}}{4}\right)\left(\Gamma_{i}^{\frac{1}{2}}-\frac{(n-1)^{2}}{6}\right)
$$

That paper motivated the present one, where we treat the clamped plate problem on a bounded domain of a noncompact simply connected complete Riemannian manifold $M^{n}$.

Theorem 1.2. Assume that $M^{n}(n \geq 3)$ is a noncompact simply connected complete Riemannian manifold with sectional curvature Sec satisfying $-K^{2} \leq \operatorname{Sec} \leq-k^{2}$, where $K \geq k \geq 0$ are constants. For a bounded domain $\Omega$ in $M$, let $\Gamma_{i}$ be the $i$-th 
eigenvalue of the eigenvalue problem (1-2). Then we have

$$
\begin{aligned}
& \sum_{i=1}^{k}\left(\Gamma_{k+1}-\Gamma_{i}\right)^{2} \leq 24 \sum_{i=1}^{k}\left(\Gamma_{k+1}\right.\left.-\Gamma_{i}\right)\left(\Gamma_{i}^{\frac{1}{2}}-\frac{n-1}{4}\left((n-1) k^{2}-2\left(K^{2}-k^{2}\right)\right)\right) \\
& \times\left(\Gamma_{i}^{\frac{1}{2}}-\frac{n-1}{6}\left((n-1) k^{2}-2\left(K^{2}-k^{2}\right)\right)\right) .
\end{aligned}
$$

Remark. If $k=K=1$, that is, $M^{n}$ is a hyperbolic space $\mathbb{M}^{n}(-1)$, then the eigenvalue inequality (1-12) agrees with (1-11) obtained by Cheng and Yang. Wang and Xia [2011] generalized (1-11) under the assumption that there exists some function whose norm of gradient is 1 and whose Laplacian is a constant.

From Theorem 1.2, we can immediately obtain the following.

Corollary 1.3. Let $\Gamma_{i}$ be the $i$-th eigenvalue of the eigenvalue problem (1-2). Then we have

$$
\sum_{i=1}^{k}\left(\Gamma_{k+1}-\Gamma_{i}\right)^{2} \leq 24 \sum_{i=1}^{k}\left(\Gamma_{k+1}-\Gamma_{i}\right)\left(\Gamma_{i}-\frac{(n-1)^{2}}{16}\left((n-1) k^{2}-2\left(K^{2}-k^{2}\right)\right)^{2}\right) .
$$

\section{Preliminaries}

Let $B$ and $C$ be $(n-1) \times(n-1)$ real symmetrical matrixes. If all the eigenvalues of $B$ are equal or greater than all the ones of $C$, then we write $B \succ C$.

Let $(M, g)$ be an $n$-dimensional Riemannian manifold and $D$ the Riemannian connection. The curvature tensor is a (1,3)-tensor defined by

$$
R(X, Y) Z=D_{X} D_{Y} Z-D_{Y} D_{X} Z-D_{[X, Y]} Z
$$

for all $X, Y, Z \in \Gamma(\mathrm{TM})$. Let $\gamma:[0, b) \longrightarrow M$ be the minimal normal geodesic and $\left\{e_{i}(t)\right\}_{i=1}^{n}$ parallel orthonormal frame fields along $\gamma(t)$ such that $e_{n}(t)=\gamma(t)$. Let

$$
J_{i}(t)=\sum_{j=1}^{n-1} f_{i j}(t) e_{j}(t), i=1, \ldots, n-1,
$$

be the normal Jacobi fields along the geodesic $\gamma(t)$; that is

$$
\ddot{f}_{i j}-f_{i l} R_{n j n l}=0, \quad f_{i j}(0)=0, \quad \dot{f}_{i j}(0)=\delta_{i j},
$$

where

$$
\dot{f}_{i j}=\frac{\mathrm{d}}{\mathrm{d} t} f_{i j}(t), \quad \ddot{f}_{i j}=\frac{\mathrm{d}^{2}}{\mathrm{~d} t^{2}} f_{i j}(t), \quad R_{n j n l}=g\left(R\left(e_{n}, e_{l}\right) e_{n}, e_{j}\right)=R_{n l n j} .
$$

Set

$$
f(t)=\left(f_{i j}(t)\right)_{(n-1) \times(n-1)}, \quad K(t)=\left(R_{n l n j}(\gamma(t))\right)_{(n-1) \times(n-1)},
$$


where $f_{i j}(t)$ is on column $j$ and row $i$. Then (2-2) can be written as

$$
\left\{\begin{array}{l}
\ddot{f}(t)-f(t) K(t)=0, \quad 0<t<b \\
f(0)=0 \\
\dot{f}(0)=I_{n-1}
\end{array}\right.
$$

where $I_{n-1}$ is the $(n-1) \times(n-1)$ unit matrix.

Define the distance function $r(x)=\operatorname{distance}(x, \gamma(0))$. Then

$$
\text { Hess } r(\gamma(t))=f(t)^{-1} \dot{f}(t), \quad \Delta r(\gamma(t))=\operatorname{tr}\left(f(t)^{-1} \dot{f}(t)\right) .
$$

Assume that $\Omega$ is a bounded domain in an $n$-dimensional noncompact simply connected complete Riemannian manifold $(M, g)$ with section curvature Sec satisfying $-K^{2} \leq \operatorname{Sec} \leq-k^{2}$, where $0 \leq k \leq K$ are constants. For $p \notin \bar{\Omega}$ fixed, define the distance function $r(x)=$ distance $(x, p)$. Then from the Hessian comparison theorem (cf. [Wu et al. 1989]), we have

$$
K \frac{\cosh K r}{\sinh K r} I_{n-1} \succ \operatorname{Hess} r \succ k \frac{\cosh k r}{\sinh k r} I_{n-1} .
$$

From (2-4) and (2-5), we have

$$
(n-1) k \frac{\cosh k r}{\sinh k r} \leq \Delta r \leq(n-1) K \frac{\cosh K r}{\sinh K r} .
$$

Since $\partial_{r} \Delta r=-|\operatorname{Hess} r|^{2}-\operatorname{Ric}\left(\partial_{r}, \partial_{r}\right)$ (cf. [Petersen 1998]), we have

$$
-\partial_{r} \Delta r \leq(n-1) K^{2} \frac{\cosh ^{2} K r}{\sinh ^{2} K r}-(n-1) k^{2} .
$$

\section{Proof of Theorem 1.1}

Theorem 3.1 [Cheng and Yang 2006b]. Let $\lambda_{i}$ be the $i$-th eigenvalue of the above eigenvalue problem (1-1) and $u_{i}$ the orthonormal eigenfunction corresponding to $\lambda_{i}$; that is, $u_{i}$ satisfies

$$
\left\{\begin{aligned}
u_{i} & =-\lambda u_{i} & & \text { in } \Omega, \\
u & =0 & & \text { on } \partial \Omega, \\
\int_{\Omega} u_{i} u_{j} & =\delta_{i j} & & \text { for all } i, j=1,2, \ldots
\end{aligned}\right.
$$

Then for any $f \in C^{3}(\Omega) \cap C^{2}(\partial \Omega)$, we have

$$
\sum_{i=1}^{k}\left(\lambda_{k+1}-\lambda_{i}\right)^{2} \int_{\Omega}|\nabla f|^{2} u_{i}^{2} \leq \sum_{i=1}^{k}\left(\lambda_{k+1}-\lambda_{i}\right) \int_{\Omega}\left(2 \nabla f \cdot \nabla u_{i}+u_{i} \Delta f\right)^{2} .
$$


Proof of Theorem 1.1. Taking $f=r$ in the formula (3-1), we have

$$
\sum_{i=1}^{k}\left(\lambda_{k+1}-\lambda_{i}\right)^{2} \int_{\Omega}|\nabla r|^{2} u_{i}^{2} \leq \sum_{i=1}^{k}\left(\lambda_{k+1}-\lambda_{i}\right) \int_{\Omega}\left(2 \nabla r \cdot \nabla u_{i}+u_{i} \Delta r\right)^{2} .
$$

Since $|\nabla r|=1$, we have

$$
\sum_{i=1}^{k}\left(\lambda_{k+1}-\lambda_{i}\right)^{2} \leq \sum_{i=1}^{k}\left(\lambda_{k+1}-\lambda_{i}\right) \int_{\Omega}\left(2 \nabla r \cdot \nabla u_{i}+u_{i} \Delta r\right)^{2} .
$$

From (2-6) and (2-7), we obtain

$$
\begin{aligned}
& \int_{\Omega}\left(2 \nabla r \cdot \nabla u_{i}+u_{i} \Delta r\right)^{2} \\
& \quad=4 \int_{\Omega}\left(\nabla r \cdot \nabla u_{i}\right)^{2}+4 \int_{\Omega} u_{i} \Delta r \nabla r \cdot \nabla u_{i}+\int_{\Omega}\left(u_{i} \Delta r\right)^{2} \\
& \quad \leq 4 \int_{\Omega}\left|\nabla u_{i}\right|^{2}-\int_{\Omega} u_{i}^{2}(\Delta r)^{2}-2 \int_{\Omega} u_{i}^{2} \nabla r \cdot \nabla \Delta r \\
& =4 \int_{\Omega}\left|\nabla u_{i}\right|^{2}-\int_{\Omega} u_{i}^{2}(\Delta r)^{2}-2 \int_{\Omega} u_{i}^{2} \partial_{r} \Delta r \\
& =4 \int_{\Omega}\left|\nabla u_{i}\right|^{2}-\int_{\Omega} u_{i}^{2}(\Delta r)^{2}+2 \int_{\Omega} u_{i}^{2}\left(\operatorname{Ric}\left(\partial_{r}, \partial_{r}\right)+|\operatorname{Hess} r|^{2}\right) \\
& \leq 4 \lambda_{i}-(n-1)^{2} k^{2} \int_{\Omega} u_{i}^{2} \frac{\cosh ^{2} k r}{\sinh ^{2} k r} \\
& \quad-2(n-1) k^{2}+2(n-1) K^{2} \int_{\Omega} u_{i}^{2} \frac{\cosh ^{2} K r}{\sinh ^{2} K r} \\
& =4 \lambda_{i}-(n-1)^{2} k^{2}+2(n-1)\left(K^{2}-k^{2}\right) \\
& \quad-(n-1)^{2} \int_{\Omega} \frac{k^{2}}{\sinh ^{2} k r} u_{i}^{2}+2(n-1) \int_{\Omega} \frac{K^{2}}{\sinh ^{2} K r} u_{i}^{2} .
\end{aligned}
$$

Since $K \geq k \geq 0$ and $r>0$, we have

$$
\frac{K}{\sinh K r} \leq \frac{k}{\sinh k r} .
$$

Since $n \geq 3$, we have

$$
(n-1)^{2} \frac{k^{2}}{\sinh ^{2} k r}-2(n-1) \frac{K^{2}}{\sinh ^{2} K r} \geq(n-1)(n-3) \frac{k^{2}}{\sinh ^{2} k r} \geq 0 .
$$

Finally, we have

$$
\sum_{i=1}^{k}\left(\lambda_{k+1}-\lambda_{i}\right)^{2} \leq \sum_{i=1}^{k}\left(\lambda_{k+1}-\lambda_{i}\right)\left(4 \lambda_{i}-(n-1)^{2} k^{2}+2(n-1)\left(K^{2}-k^{2}\right)\right) .
$$




\section{Proof of Theorem 1.2}

Let $u_{i}$ be the $i$-th orthonormal eigenfunction corresponding to the eigenvalue $\Gamma_{i}$, $i=1, \ldots, k$; that is,

$$
\begin{cases}\Delta^{2} u_{i}=\Gamma_{i} u_{i} & \text { in } \Omega, \\ u_{i}=\frac{\partial u_{i}}{\partial v}=0 & \text { on } \partial \Omega \\ \int_{\Omega} u_{i} u_{j}=\delta_{i j} & \text { for any } i, j .\end{cases}
$$

Defining the functions

$$
\phi_{i}=r u_{i}-\sum_{j=1}^{k} a_{i j} u_{j}
$$

where

$$
a_{i j}=\int_{\Omega} r u_{i} u_{j}
$$

we have

$$
\left.\phi_{i}\right|_{\partial \Omega}=\left.\frac{\partial \phi_{i}}{\partial v}\right|_{\partial \Omega}=0 \quad \text { and } \quad \int_{\Omega} \phi_{i} u_{j}=0 \quad \text { for all } i, j=1, \ldots, k .
$$

Therefore, we know that $\phi_{i} \mathrm{~s}$ are trial functions. From the Rayleigh-Ritz inequality [Chavel 1984], we have

$$
\Gamma_{k+1} \leq \frac{1}{\left\|\phi_{i}\right\|^{2}} \int_{\Omega}\left(\Delta \phi_{i}\right)^{2}
$$

where

$$
\left\|\phi_{i}\right\|^{2}=\int_{\Omega} \phi_{i}^{2}
$$

From (4-1) and (4-2), we have

$$
\begin{aligned}
\Gamma_{k+1} \int_{\Omega} \phi_{i}^{2} & \leq \int_{\Omega}\left(\Delta \phi_{i}\right)^{2}=\int_{\Omega} \phi_{i} \Delta^{2} \phi_{i}=\int_{\Omega} \phi_{i} \Delta^{2}\left(r u_{i}-\sum_{j=1}^{k} a_{i j} u_{j}\right) \\
& =\int_{\Omega} \phi_{i} \Delta^{2}\left(r u_{i}\right)=\int_{\Omega} \phi_{i}\left(\Delta^{2}\left(r u_{i}\right)-\Gamma_{i} r u_{i}\right)+\Gamma_{i} \int_{\Omega} \phi_{i}^{2},
\end{aligned}
$$

that is,

$$
\left(\Gamma_{k+1}-\Gamma_{i}\right)\left\|\phi_{i}\right\|^{2} \leq \int_{\Omega} \phi_{i}\left(\Delta^{2}\left(r u_{i}\right)-\Gamma_{i} r u_{i}\right)
$$


From the definition of $\phi_{i}$ and (4-2), we have

$$
\begin{aligned}
\left(\Gamma_{k+1}\right. & \left.-\Gamma_{i}\right)\left\|\phi_{i}\right\|^{2} \\
& \leq \int_{\Omega}\left(r u_{i}-\sum_{j=1}^{k} a_{i j} u_{j}\right)\left(\Delta^{2}\left(r u_{i}\right)-\Gamma_{i} r u_{i}\right) \\
& =\int_{\Omega} r u_{i}\left(\Delta^{2}\left(r u_{i}\right)-\Gamma_{i} r u_{i}\right)+\sum_{j=1}^{k} a_{i j}^{2}\left(\Gamma_{i}-\Gamma_{j}\right) \\
& =\int_{\Omega} r u_{i}\left(\Delta\left(u_{i} \Delta r\right)+2 \Delta\left(\nabla r \cdot \nabla u_{i}\right)+2 \nabla r \cdot \nabla \Delta u_{i}+\Delta r \Delta u_{i}\right) \\
& +\sum_{j=1}^{k} a_{i j}^{2}\left(\Gamma_{i}-\Gamma_{j}\right) .
\end{aligned}
$$

From (2-6), (2-7), and Stokes' theorem, by a direct calculation, we have

$$
\begin{aligned}
& \int_{\Omega} r u_{i}\left(\Delta\left(u_{i} \Delta r\right)+2 \Delta\left(\nabla r \cdot \nabla u_{i}\right)+2 \nabla r \cdot \nabla \Delta u_{i}+\Delta r \Delta u_{i}\right) \\
& =\int_{\Omega}\left(\Delta\left(r u_{i}\right)\left(u_{i} \Delta r+2 \nabla r \cdot \nabla u_{i}\right)+u_{i} \nabla r^{2} \cdot \nabla \Delta u_{i}+u_{i} r \Delta r \Delta u_{i}\right) \\
& =\int_{\Omega}\left(\left(u_{i} \Delta r+2 \nabla r \cdot \nabla u_{i}+r \Delta u_{i}\right)\left(u_{i} \Delta r+2 \nabla r \cdot \nabla u_{i}\right)\right. \\
& \left.+u_{i} \nabla r^{2} \cdot \nabla \Delta u_{i}+u_{i} r \Delta r \Delta u_{i}\right)
\end{aligned}
$$$$
=\int_{\Omega}\left((\Delta r)^{2} u_{i}^{2}+2 \nabla r \cdot \nabla u_{i}^{2} \Delta r+4\left(\nabla r \cdot \nabla u_{i}\right)^{2}+2 r u_{i} \Delta r \Delta u_{i}+\nabla r^{2} \cdot \nabla u_{i} \Delta u_{i}\right)
$$$$
+\int_{\Omega} u_{i} \nabla r^{2} \cdot \nabla \Delta u_{i}
$$$$
=\int_{\Omega}\left((\Delta r)^{2} u_{i}^{2}+2 \nabla r \cdot \nabla u_{i}^{2} \Delta r+4\left(\nabla r \cdot \nabla u_{i}\right)^{2}+2 r u_{i} \Delta r \Delta u_{i}+\nabla r^{2} \cdot \nabla\left(u_{i} \Delta u_{i}\right)\right)
$$$$
=\int_{\Omega}\left((\Delta r)^{2} u_{i}^{2}+2 \nabla r \cdot \nabla u_{i}^{2} \Delta r+4\left(\nabla r \cdot \nabla u_{i}\right)^{2}+\left(2 r \Delta r-\Delta r^{2}\right) u_{i} \Delta u_{i}\right)
$$$$
=\int_{\Omega}\left(-(\Delta r)^{2} u_{i}^{2}-2 u_{i}^{2} \nabla r \cdot \nabla \Delta r+4\left(\nabla r \cdot \nabla u_{i}\right)^{2}-2 u_{i} \Delta u_{i}\right)
$$$$
\leq \int_{\Omega}\left(4\left|\nabla u_{i}\right|^{2}-2 u_{i} \Delta u_{i}\right)-\int_{\Omega} u_{i}^{2}\left(2 \nabla r \cdot \nabla \Delta r+(\Delta r)^{2}\right)
$$$$
\leq \int_{\Omega} u_{i}^{2}\left(-(n-1)^{2} k^{2} \frac{\cosh ^{2} k r}{\sinh ^{2} k r}-2(n-1) k^{2}+2(n-1) K^{2} \frac{\cosh ^{2} K r}{\sinh ^{2} K r}\right)
$$$$
+\int_{\Omega}\left(4\left|\nabla u_{i}\right|^{2}+2 u_{i}\left(-\Delta u_{i}\right)\right)
$$ 
Since $n \geq 3$, from (3-4), we have

$$
\begin{aligned}
\int_{\Omega} r u_{i}\left(\Delta\left(u_{i} \Delta r\right)\right. & \left.+2 \Delta\left(\nabla r \cdot \nabla u_{i}\right)+2 \nabla r \cdot \nabla \Delta u_{i}+\Delta r \Delta u_{i}\right) \\
\leq & 6 \int_{\Omega} u_{i}\left(-\Delta u_{i}\right)-\int_{\Omega} u_{i}^{2}\left((n-1)^{2} k^{2}-2(n-1)\left(K^{2}-k^{2}\right)\right) \\
\leq & 6\left(\int_{\Omega}\left(\Delta u_{i}\right)^{2}\right)^{\frac{1}{2}}-(n-1)\left((n-1) k^{2}-2\left(K^{2}-k^{2}\right)\right) \\
= & 6\left(\Gamma_{i}^{\frac{1}{2}}-\frac{n-1}{6}\left((n-1) k^{2}-2\left(K^{2}-k^{2}\right)\right)\right) .
\end{aligned}
$$

From (4-4) and (4-6), we deduce

$$
\begin{aligned}
& \left(\Gamma_{k+1}-\Gamma_{i}\right)\left\|\phi_{i}\right\|^{2} \\
& \quad \leq 6\left(\Gamma_{i}^{\frac{1}{2}}-\frac{n-1}{6}\left((n-1) k^{2}-2\left(K^{2}-k^{2}\right)\right)\right)+\sum_{j=1}^{k} a_{i j}^{2}\left(\Gamma_{i}-\Gamma_{j}\right)
\end{aligned}
$$

Defining

$$
b_{i j}=\int_{\Omega}\left(\nabla r \cdot \nabla u_{i}+\frac{1}{2} u_{i} \Delta r\right) u_{j}
$$

we have

$$
b_{i j}=-b_{j i}
$$

From the definitions of $b_{i j}$ and $\phi_{i}$, we obtain

$$
\begin{aligned}
-2 \int_{\Omega} \phi_{i}\left(\nabla r \cdot \nabla u_{i}+\frac{1}{2} \Delta r\right. & \left.u_{i}\right) \\
& =-2 \int_{\Omega}\left(r u_{i}-\sum_{j=1}^{k} a_{i j} u_{j}\right)\left(\nabla r \cdot \nabla u_{i}+\frac{1}{2} \Delta r u_{i}\right) \\
& =-2 \int_{\Omega} r u_{i}\left(\nabla r \cdot \nabla u_{i}+\frac{1}{2} \Delta r u_{i}\right)+2 \sum_{j=1}^{k} a_{i j} b_{i j} \\
& =-\int_{\Omega}\left(\frac{1}{2} \nabla r^{2} \cdot \nabla u_{i}^{2}+r \Delta r u_{i}^{2}\right)+2 \sum_{j=1}^{k} a_{i j} b_{i j} \\
& =1+2 \sum_{j=1}^{k} a_{i j} b_{i j}
\end{aligned}
$$


From (4-2), (4-8), and the Cauchy-Schwartz inequality, we have

$$
\begin{aligned}
1+2 \sum_{j=1}^{k} a_{i j} b_{i j} & =-2 \int_{\Omega} \phi_{i}\left(\nabla r \cdot \nabla u_{i}+\frac{1}{2} u_{i} \Delta r\right) \\
& =-2 \int_{\Omega} \phi_{i}\left(\nabla r \cdot \nabla u_{i}+\frac{1}{2} u_{i} \Delta r-\sum_{j=1}^{k} b_{i j} u_{j}\right) \\
& \leq \alpha_{i}\left\|\phi_{i}\right\|^{2}+\frac{1}{\alpha_{i}}\left\|\nabla r \cdot \nabla u_{i}+\frac{1}{2} u_{i} \Delta r-\sum_{j=1}^{k} b_{i j} u_{j}\right\|^{2} \\
& =\alpha_{i}\left\|\phi_{i}\right\|^{2}+\frac{1}{\alpha_{i}}\left(\left\|\nabla r \cdot \nabla u_{i}+\frac{1}{2} u_{i} \Delta r\right\|^{2}-\sum_{j=1}^{k} b_{i j}^{2}\right),
\end{aligned}
$$

where $\alpha_{i}>0$ is a positive constant.

If $\Gamma_{k+1}-\Gamma_{i}>0$, defining

$$
\alpha_{i}=\left(\Gamma_{k+1}-\Gamma_{i}\right) \beta_{i} \quad \text { for } \beta_{i}>0,
$$

we infer that

$$
\begin{aligned}
&(4-10) \quad\left(\Gamma_{k+1}-\right.\left.\Gamma_{i}\right)^{2}\left(1+2 \sum_{j=1}^{k} a_{i j} b_{i j}\right) \\
& \leq\left(\Gamma_{k+1}-\Gamma_{i}\right)^{3} \beta_{i}\left\|\phi_{i}\right\|^{2} \\
& \quad+m \frac{1}{\beta_{i}}\left(\Gamma_{k+1}-\Gamma_{i}\right)\left(\left\|\nabla r \cdot \nabla u_{i}+\frac{1}{2} u_{i} \Delta r\right\|^{2}-\sum_{j=1}^{k} b_{i j}^{2}\right) .
\end{aligned}
$$

From (2-6), (2-7), and (3-4), we obtain

$$
\begin{aligned}
\int_{\Omega}\left(2 \nabla r \cdot \nabla u_{i}+u_{i}\right. & \Delta r)^{2} \\
& =4 \int_{\Omega}\left(\nabla r \cdot \nabla u_{i}\right)^{2}+4 \int_{\Omega} u_{i} \Delta r \nabla r \cdot \nabla u_{i}+\int_{\Omega}\left(u_{i} \Delta r\right)^{2} \\
& \leq 4 \int_{\Omega}\left|\nabla u_{i}\right|^{2}-\int_{\Omega} u_{i}^{2}(\Delta r)^{2}-2 \int_{\Omega} u_{i}^{2} \nabla r \cdot \nabla \Delta r \\
& \leq 4 \Gamma_{i}^{\frac{1}{2}}-(n-1)\left((n-1) k^{2}-2\left(K^{2}-k^{2}\right)\right) \\
& \leq 4\left(\Gamma_{i}^{\frac{1}{2}}-\frac{n-1}{4}\left((n-1) k^{2}-2\left(K^{2}-k^{2}\right)\right)\right) .
\end{aligned}
$$


Therefore, from (4-7), (4-9), (4-10), and (4-11), we obtain

$$
\begin{aligned}
& \left(\Gamma_{k+1}-\Gamma_{i}\right)^{2}\left(1+2 \sum_{j=1}^{k} a_{i j} b_{i j}\right) \\
& \leq\left(\Gamma_{k+1}-\Gamma_{i}\right)^{2} \beta_{i}\left(6\left(\Gamma_{i}^{\frac{1}{2}}-\frac{n-1}{6}\left((n-1) k^{2}-2\left(K^{2}-k^{2}\right)\right)\right)+\sum_{j=1}^{k} a_{i j}^{2}\left(\Gamma_{i}-\Gamma_{j}\right)\right) \\
& +\frac{1}{\beta_{i}}\left(\Gamma_{k+1}-\Gamma_{i}\right)\left(\Gamma_{i}^{\frac{1}{2}}-\frac{n-1}{4}\left((n-1) k^{2}-2\left(K^{2}-k^{2}\right)\right)\right) \\
& -\frac{1}{\beta_{i}}\left(\Gamma_{k+1}-\Gamma_{i}\right) \sum_{j=1}^{k} b_{i j}^{2} .
\end{aligned}
$$

From the antisymmetry of $b_{i j}$ and the Cauchy-Schwartz inequality, we have

$$
\begin{aligned}
2 \sum_{i, j=1}^{k}\left(\Gamma_{k+1}-\Gamma_{i}\right)^{2} a_{i j} b_{i j} & \\
& -\sum_{i, j=1}^{k}\left(\Gamma_{k+1}-\Gamma_{i}\right)\left(\Gamma_{i}-\Gamma_{j}\right)^{2} \beta_{i} a_{i j}^{2}-\sum_{i, j=1}^{k} \frac{1}{\beta_{i}}\left(\Gamma_{k+1}-\Gamma_{i}\right) b_{i j}^{2} \leq 0 .
\end{aligned}
$$

From the above inequality and (4-12), we obtain

$$
\begin{aligned}
& \sum_{i=1}^{k}\left(\Gamma_{k+1}-\Gamma_{i}\right)^{2} \\
& \leq 6 \sum_{i=1}^{k}\left(\Gamma_{k+1}-\Gamma_{i}\right)^{2} \beta_{i}\left(\Gamma_{i}^{\frac{1}{2}}-\frac{n-1}{6}\left((n-1) k^{2}-2\left(K^{2}-k^{2}\right)\right)\right) \\
& \quad+\sum_{i=1}^{k} \frac{1}{\beta_{i}}\left(\Gamma_{k+1}-\Gamma_{i}\right)\left(\Gamma_{i}^{\frac{1}{2}}-\frac{n-1}{4}\left((n-1) k^{2}-2\left(K^{2}-k^{2}\right)\right)\right) \\
& \quad+\sum_{i, j=1}^{k}\left(\Gamma_{k+1}-\Gamma_{i}\right)\left(\Gamma_{k+1}-\Gamma_{j}\right)\left(\Gamma_{i}-\Gamma_{j}\right) \beta_{i} a_{i j}^{2} .
\end{aligned}
$$

From the variational principle, we can prove that

$$
\Gamma_{i} \geq \lambda_{i}^{2}
$$

where $\lambda_{i}$ denotes the $i$-th eigenvalue of the Dirichlet eigenvalue problem of the Laplacian on the same domain $\Omega$. Since $4 \lambda_{1} \geq(n-1)^{2} k^{2}-2\left(K^{2}-k^{2}\right)$ from (3-2), setting

$$
\beta_{i}=\beta\left(\Gamma_{i}^{\frac{1}{2}}-\frac{n-1}{6}\left((n-1) k^{2}-2\left(K^{2}-k^{2}\right)\right)\right)^{-1} \text { for } \beta>0
$$


gives us

$$
\begin{aligned}
\sum_{i, j=1}^{k}\left(\Gamma_{k+1}-\Gamma_{i}\right) & \left(\Gamma_{k+1}-\Gamma_{j}\right)\left(\Gamma_{i}-\Gamma_{j}\right) \beta_{i} a_{i j}^{2} \\
& =\frac{1}{2} \sum_{i, j=1}^{k}\left(\Gamma_{k+1}-\Gamma_{i}\right)\left(\Gamma_{k+1}-\Gamma_{j}\right)\left(\Gamma_{i}-\Gamma_{j}\right)\left(\beta_{i}-\beta_{j}\right) a_{i j}^{2} \\
& =-\frac{1}{2} \beta \sum_{i, j=1}^{k} \frac{\left(\Gamma_{k+1}-\Gamma_{i}\right)\left(\Gamma_{k+1}-\Gamma_{j}\right)\left(\Gamma_{i}-\Gamma_{j}\right)\left(\Gamma_{i}^{\frac{1}{2}}-\Gamma_{j}^{\frac{1}{2}}\right)}{\left(\Gamma_{i}^{\frac{1}{2}}-\frac{n-1}{6}\left((n-1) k^{2}-2\left(K^{2}-k^{2}\right)\right)\right)} a_{i j}^{2} \\
& \times\left(\Gamma_{i}^{\frac{1}{2}}-\frac{n-1}{6}\left((n-1) k^{2}-2\left(K^{2}-k^{2}\right)\right)\right) \\
& \leq 0 .
\end{aligned}
$$

Therefore, we obtain

$$
\begin{aligned}
& \sum_{i=1}^{k}\left(\Gamma_{k+1}-\right.\left.\Gamma_{i}\right)^{2} \leq 6 \beta \sum_{i=1}^{k}\left(\Gamma_{k+1}-\Gamma_{i}\right)^{2} \\
&+\frac{1}{\beta} \sum_{i=1}^{k}\left(\Gamma_{k+1}-\Gamma_{i}\right)\left(\Gamma_{i}^{\frac{1}{2}}-\frac{n-1}{4}\left((n-1) k^{2}-2\left(K^{2}-k^{2}\right)\right)\right) . \\
& \quad \times\left(\Gamma_{i}^{\frac{1}{2}}-\frac{n-1}{6}\left((n-1) k^{2}-2\left(K^{2}-k^{2}\right)\right)\right) .
\end{aligned}
$$

Finally, taking $\beta=\frac{1}{12}$, we deduce (1-12). This completes the proof of Theorem 1.2.

\section{Acknowledgements}

We thank Professor Hongcang Yang for his encouragement, suggestions, and support. We would also like to thank the referee for helpful comments and for bringing [Wang and Xia 2011] to our attention.

\section{References}

[Ashbaugh 1999] M. S. Ashbaugh, "Isoperimetric and universal inequalities for eigenvalues", pp. 95-139 in Spectral theory and geometry (Edinburgh, 1998), edited by E. B. Davies and Y. Safarov, London Math. Soc. Lecture Note Ser. 273, Cambridge University Press, Cambridge, 1999. MR 2001a:35131 Zbl 0937.35114

[Ashbaugh 2002] M. S. Ashbaugh, "The universal eigenvalue bounds of Payne-Pólya-Weinberger, Hile-Protter, and H. C. Yang: spectral and inverse spectral theory", Proc. Indian Acad. Sci. (Math. Sci.) 112:1 (2002), 3-30. MR 2004c:35302 Zbl 1199.35261

[Ashbaugh and Hermi 2007] M. S. Ashbaugh and L. Hermi, "On Harrell-Stubbe type inequalities for the discrete spectrum of a self-adjoint operator", preprint, 2007. arXiv 0712.4396

[Chavel 1984] I. Chavel, Eigenvalues in Riemannian geometry, Pure and Applied Mathematics 115, Academic Press, Orlando, FL, 1984. MR 86g:58140 Zbl 0551.53001 
[Chen and Cheng 2008] D. Chen and Q.-M. Cheng, "Extrinsic estimates for eigenvalues of the Laplace operator”, J. Math. Soc. Japan 60:2 (2008), 325-339. MR 2010b:35323 Zbl 1147.35060

[Chen and Qian 1990] Z. C. Chen and C. L. Qian, "Estimates for discrete spectrum of Laplacian operator with any order", J. China Univ. Sci. Tech. 20:3 (1990), 259-266. MR 92c:35087 Zbl 0748.35022

[Cheng and Yang 2005] Q.-M. Cheng and H. C. Yang, "Estimates on eigenvalues of Laplacian", Math. Ann. 331:2 (2005), 445-460. MR 2005i:58038 Zbl 1122.35086

[Cheng and Yang 2006a] Q.-M. Cheng and H. C. Yang, "Inequalities for eigenvalues of a clamped plate problem", Trans. Amer. Math. Soc. 358:6 (2006), 2625-2635. MR 2006m:35263 Zbl 1096. 35095

[Cheng and Yang 2006b] Q.-M. Cheng and H. C. Yang, "Inequalities for eigenvalues of Laplacian on domains and compact complex hypersurfaces in complex projective spaces", J. Math. Soc. Japan 58:2 (2006), 545-561. MR 2007k:58051 Zbl 1127.35026

[Cheng and Yang 2007] Q.-M. Cheng and H. C. Yang, "Bounds on eigenvalues of Dirichlet Laplacian”, Math. Ann. 337:1 (2007), 159-175. MR 2007k:35064 Zbl 1110.35052

[Cheng and Yang 2009] Q.-M. Cheng and H. C. Yang, "Estimates for eigenvalues on Riemannian manifolds", J. Differential Equations 247:8 (2009), 2270-2281. MR 2010j:58066 Zbl 1180.35390

[Cheng and Yang 2011] Q.-M. Cheng and H. C. Yang, "Universal inequalities for eigenvalues of a clamped plate problem on a hyperbolic space", Proc. Amer. Math. Soc. 139:2 (2011), 461-471. MR 2012b:35234 Zbl 1209.35089

[Cheng et al. 2010] Q.-M. Cheng, T. Ichikawa, and S. Mametsuka, "Estimates for eigenvalues of a clamped plate problem on Riemannian manifolds", J. Math. Soc. Japan 62:2 (2010), 673-686. MR 2011e:58039 Zbl 1191.35192

[El Soufi et al. 2009] A. El Soufi, E. M. Harrell, II, and S. Ilias, "Universal inequalities for the eigenvalues of Laplace and Schrödinger operators on submanifolds", Trans. Amer. Math. Soc. 361:5 (2009), 2337-2350. MR 2010e:58032 Zbl 1162.58009

[Harrell 2007] E. M. Harrell, II, "Commutators, eigenvalue gaps, and mean curvature in the theory of Schrödinger operators”, Comm. Partial Differential Equations 32:3 (2007), 401-413. MR 2008i: $35041 \mathrm{Zbl} 05150097$

[Harrell and Michel 1994] E. M. Harrell, II and P. L. Michel, "Commutator bounds for eigenvalues, with applications to spectral geometry", Comm. Partial Differential Equations 19:11-12 (1994), 2037-2055. MR 95i:58182 Zbl 0815.35078

[Hile and Protter 1980] G. N. Hile and M. H. Protter, "Inequalities for eigenvalues of the Laplacian", Indiana Univ. Math. J. 29:4 (1980), 523-538. MR 82c:35052 Zbl 0454.35064

[Hile and Yeh 1984] G. N. Hile and R. Z. Yeh, "Inequalities for eigenvalues of the biharmonic operator”, Pacific J. Math. 112:1 (1984), 115-133. MR 85k:35170 Zbl 0541.35059

[Hook 1990] S. M. Hook, "Domain-independent upper bounds for eigenvalues of elliptic operators", Trans. Amer. Math. Soc. 318:2 (1990), 615-642. MR 90h:35075 Zbl 0727.35096

[Payne et al. 1955] L. E. Payne, G. Pólya, and H. F. Weinberger, "Sur le quotient de deux fréquences propres consécutives”, C. R. Acad. Sci. Paris 241 (1955), 917-919. MR 17,372d Zbl 0065.08801

[Payne et al. 1956] L. E. Payne, G. Pólya, and H. F. Weinberger, "On the ratio of consecutive eigenvalues”, J. Math. and Phys. 35 (1956), 289-298. MR 18,905c Zbl 0073.08203

[Petersen 1998] P. Petersen, Riemannian geometry, vol. 171, Grad. Texts in Math., Springer, New York, 1998. 2nd ed. published in 2006. MR 98m:53001 Zbl 0914.53001 
[Protter 1988] M. H. Protter, "Universal inequalities for eigenvalues", pp. 111-120 in Maximum principles and eigenvalue problems in partial differential equations (Knoxville, TN, 1987), edited by P. W. Schaefer, Pitman Res. Notes Math. Ser. 175, Longman Scientific \& Technical, Harlow, 1988. MR 89k:35167 Zbl 0663.35052

[Wang and Xia 2007] Q. Wang and C. Xia, "Universal bounds for eigenvalues of the biharmonic operator on Riemannian manifolds", J. Funct. Anal. 245:1 (2007), 334-352. MR 2008e:58033 Zbl 1113.58013

[Wang and Xia 2011] Q. Wang and C. Xia, "Inequalities for eigenvalues of a clamped plate problem”, Calc. Var. Partial Differential Equations 40:1-2 (2011), 273-289. MR 2012a:35215 Zbl 1205. 35175

[Wu et al. 1989] H. Wu, C. L. Shen, and Y. L. Yu, Introduction to Riemannian Geometry, Peking University Press, Peking, 1989. In Chinese.

[Yang 1991] H. C. Yang, "An estimate of the difference between consecutive eigenvalues", preprint IC/91/60, International Centre for Theoretical Physics (ICTP), Trieste, 1991.

Received January 18, 2011. Revised October 26, 2011.

\section{DAGUANG CHEN}

DEPARTMENT OF MATHEMATICAL SCIENCES

TSINGHUA UNIVERSITY

BEIJING, 100084

CHINA

dgchen@math.tsinghua.edu.cn

TAO ZHENG

Hua LoO-Keng Key Laboratory of Mathematics

ChInese ACADEMY OF SCIENCES

BEIJING, 100190

CHINA

zhengtao08@mails.gucas.ac.cn

MIN LU

Department of Applied Mathematics

NANJING AUdit UNIVERSITY

NANJING, 210029

CHINA

luminm@foxmail.com 


\title{
PACIFIC JOURNAL OF MATHEMATICS
}

\author{
http://pacificmath.org \\ Founded in 1951 by \\ E. F. Beckenbach (1906-1982) and F. Wolf (1904-1989)
}

\section{EDITORS}

V. S. Varadarajan (Managing Editor)

Department of Mathematics

University of California

Los Angeles, CA 90095-1555

pacific@math.ucla.edu

Vyjayanthi Chari

Department of Mathematics

University of California

Riverside, CA 92521-0135

chari@math.ucr.edu

\section{Robert Finn}

Department of Mathematics Stanford University

Stanford, CA 94305-2125

finn@math.stanford.edu

Kefeng Liu

Department of Mathematics

University of California

Los Angeles, CA 90095-1555

liu@math.ucla.edu
Darren Long

Department of Mathematics

University of California

Santa Barbara, CA 93106-3080

long@math.ucsb.edu

Jiang-Hua Lu

Department of Mathematics

The University of Hong Kong

Pokfulam Rd., Hong Kong jhlu@maths.hku.hk

Alexander Merkurjev

Department of Mathematics

University of California

Los Angeles, CA 90095-1555

merkurev@math.ucla.edu
Sorin Popa

Department of Mathematics University of California

Los Angeles, CA 90095-1555 popa@math.ucla.edu

Jie Qing

Department of Mathematics

University of California

Santa Cruz, CA 95064

qing@cats.ucsc.edu

Jonathan Rogawski

Department of Mathematics

University of California

Los Angeles, CA 90095-1555

jonr@math.ucla.edu

\section{PRODUCTION}

pacific@math.berkeley.edu

\section{SUPPORTING INSTITUTIONS}

ACADEMIA SINICA, TAIPEI

CALIFORNIA INST. OF TECHNOLOGY INST. DE MATEMÁTICA PURA E APLICADA KEIO UNIVERSITY

MATH. SCIENCES RESEARCH INSTITUTE NEW MEXICO STATE UNIV.

OREGON STATE UNIV.

\author{
STANFORD UNIVERSITY \\ UNIV. OF BRITISH COLUMBIA \\ UNIV. OF CALIFORNIA, BERKELEY \\ UNIV. OF CALIFORNIA, DAVIS \\ UNIV. OF CALIFORNIA, LOS ANGELES \\ UNIV. OF CALIFORNIA, RIVERSIDE \\ UNIV. OF CALIFORNIA, SAN DIEGO \\ UNIV. OF CALIF., SANTA BARBARA
}

\author{
UNIV. OF CALIF., SANTA CRUZ \\ UNIV. OF MONTANA \\ UNIV. OF OREGON \\ UNIV. OF SOUTHERN CALIFORNIA \\ UNIV. OF UTAH \\ UNIV. OF WASHINGTON \\ WASHINGTON STATE UNIVERSITY
}

These supporting institutions contribute to the cost of publication of this Journal, but they are not owners or publishers and have no responsibility for its contents or policies.

See inside back cover or pacificmath.org for submission instructions.

The subscription price for 2012 is US \$420/year for the electronic version, and \$485/year for print and electronic.

Subscriptions, requests for back issues from the last three years and changes of subscribers address should be sent to Pacific Journal of Mathematics, P.O. Box 4163, Berkeley, CA 94704-0163, U.S.A. Prior back issues are obtainable from Periodicals Service Company, 11 Main Street, Germantown, NY 12526-5635. The Pacific Journal of Mathematics is indexed by Mathematical Reviews, Zentralblatt MATH, PASCAL CNRS Index, Referativnyi Zhurnal, Current Mathematical Publications and the Science Citation Index.

The Pacific Journal of Mathematics (ISSN 0030-8730) at the University of California, c/o Department of Mathematics, 969 Evans Hall, Berkeley, CA 94720-3840, is published monthly except July and August. Periodical rate postage paid at Berkeley, CA 94704, and additional mailing offices. POSTMASTER: send address changes to Pacific Journal of Mathematics, P.O. Box 4163, Berkeley, CA 94704-0163.

PJM peer review and production are managed by EditFLOW ${ }^{\mathrm{TM}}$ from Mathematical Sciences Publishers.

PUBLISHED BY PACIFIC JOURNAL OF MATHEMATICS

at the University of California, Berkeley 94720-3840

A NON-PROFIT CORPORATION

Typeset in LATEX

Copyright $(02012$ by Pacific Journal of Mathematics 


\section{PACIFIC JOURNAL OF MATHEMATICS}

Volume $255 \quad$ No. $1 \quad$ January 2012

Averaging sequences

FERnANDo Alcalde Cuesta and Ana Rechtman

Affine group schemes over symmetric monoidal categories

ABHISHEK BANERJEE

Eigenvalue estimates on domains in complete noncompact Riemannian

manifolds

Daguang Chen, TAO Zheng and Min LU

Realizing the local Weil representation over a number field

GERALD CLIFF and DAVID MCNEILLY

Lagrangian submanifolds in complex projective space with parallel second fundamental form

Franki DiLlen, Haizhong Li, LuC VRANCKEN and XiANFENG

WANG

Ultra-discretization of the $D_{4}^{(3)}$-geometric crystal to the $G_{2}^{(1)}$-perfect crystals

Mana Igarashi, KaIlash C. MisRa and ToshiKi NAKASHIMA Connectivity properties for actions on locally finite trees

KEITH JONES

Remarks on the curvature behavior at the first singular time of the Ricci flow

NAM Q. LE and NATASA SESUm

Stability of capillary surfaces with planar boundary in the absence of gravity

PetKo I. MARINOV

Small hyperbolic polyhedra

SHAWN RAFALSKI

Hurwitz spaces of coverings with two special fibers and monodromy group 241 a Weyl group of type $B_{d}$

FRANCESCA VETRO 